\title{
ASSESSMENT OF THE EFFECT OF REDUCED COMPOSITIONAL \\ HETEROGENEITY ON FRACTURE RESISTANCE OF HUMAN CORTICAL BONE \\ USING FINITE ELEMENT MODELING
}

\author{
Ahmet Demirtas ${ }^{1}$, Erin Curran $^{1}$, Ani Ural $^{1}$ \\ ${ }^{1}$ Department of Mechanical Engineering, Villanova University, 800 Lancaster Avenue, \\ Villanova, PA
}

Original Article

\section{Corresponding Author}

\author{
Ani Ural \\ Associate Professor \\ Department of Mechanical Engineering \\ Villanova University \\ 800 Lancaster Avenue, Villanova, PA 19085 \\ E-mail: ani.ural@villanova.edu
}

Phone:(610) 519-7735

Fax: (610) 519-7312 


\section{ABSTRACT}

2 The recent reports of atypical femoral fracture and its possible association with prolonged

3 bisphosphonate use highlighted the importance of a thorough understanding of mechanical

4 modifications in bone due to bisphosphonate treatment. The reduced compositional

5 heterogeneity is one of the modifications in bone due to extensive suppression of bone turnover.

6 Although experimental evaluations suggested that compositional changes lead to a reduction in

7 the heterogeneity of elastic properties, there is limited information on the extent of influence of

8 reduced heterogeneity on fracture resistance of cortical bone. As a result, the goal of the current

9 study is to evaluate the influence of varying the number of unique elastic and fracture properties

10 for osteons, interstitial bone, and cement lines on fracture resistance across seven different

11 human cortical bone specimens using finite element modeling. Fracture resistance of seven

12 human cortical bone samples under homogeneous and three different heterogeneous material

13 levels was evaluated using a compact tension test setup. The simulation results predicted that the

14 crack volume was the highest for the models with homogeneous material properties. Increasing

15 heterogeneity resulted in a lower amount of crack volume indicating an increase in fracture

16 resistance of cortical bone. This reduction was observed up to a certain level of heterogeneity

17 after which further beneficial effects of heterogeneity diminished suggesting a possible optimum

18 level of heterogeneity for the bone tissue. The homogeneous models demonstrated limited areas

19 of damage with extensive crack formation. On the other hand, the heterogeneity in the material

20 properties led to increased damage volume and a more variable distribution of damage compared

21 to the homogeneous models. This resulted in uncracked regions which tended to have less

22 damage accumulation preventing extensive crack propagation. The results also showed that the

23 percent osteonal area was inversely correlated with crack volume and more evenly distributed 
1 osteons led to a lower amount of crack growth for all levels of material heterogeneity. In

2 summary, this study developed a new computational modeling approach that directly evaluated

3 the influence of heterogeneity in elastic and fracture material properties on fracture resistance of

4 cortical bone. The results established new information that showed the adverse effects of reduced

5 heterogeneity on fracture resistance in cortical bone and demonstrated the nonlinear relationship

6 between heterogeneity and fracture resistance. This new computational modeling approach

7 provides a tool that can be used to improve the understanding of the effects of material level

8 changes due to prolonged BP use on the overall bone fracture behavior. It may also bring

9 additional insight into the causes of unusual fractures, such as AFF and their possible association

10 with long term BP use.

11

12 Keywords: Atypical femoral fracture; Heterogeneity; Cortical bone; Cohesive finite element

13 modeling; Bisphosphonate

14 


\section{1. INTRODUCTION}

2 Bisphosphonates (BP) are the most commonly used osteoporosis treatment that has been

3 effective in preventing osteoporotic fractures by suppressing bone turnover. The reduction in

4 bone turnover preserves the microarchitecture of the bone and maintains or increases areal bone

5 mineral density (BMD). Despite the beneficial effects of BPs, there is accumulating evidence of

6 a potential complication in the form of atypical femoral fracture (AFF) [1, 2]. AFF is a

7 catastrophic failure of the femur occurring in the subtrochanteric region and diaphysis of the

8 femur. Although the occurrence rate of AFF in the general population is quite low, it leads to

9 high rate of morbidity and mortality [2]. These fractures have different characteristics than

10 ordinary osteoporotic femoral fractures. Additionally, areal BMD is not a good predictor of AFF

11 risk [3]. Although a causal relationship between AFF and BP has not yet been shown, several

12 studies demonstrated a duration-dependent association between AFF and BP use [1, 4-7].

13 The recent reports of AFF in patients who have undergone long-term BP treatment brought

14 into attention the possibility of adverse mechanical modifications in bone due to extensive

15 suppression of bone turnover $[2,8]$. One of these modifications has been suggested as reduced

16 compositional heterogeneity of the bone [9, 10]. Studies performed on bone biopsies from

17 individuals under BP treatment have shown increased mineralization and collagen maturity as

18 well as reduced mineral and matrix heterogeneity [9-14]. Similar results were obtained on

19 biopsies from individuals with fracture history in small populations [15, 16]. On the other hand,

20 no association between fracture status and heterogeneity in mineralization and collagen maturity

21 was found in a larger age-matched cohort [17]. In addition, several studies reported increasing

22 trends in mineralization heterogeneity in fracture groups in pediatric patients [18] as well as in 
1 adults compared to nonfracture controls $[19,20]$. These results indicate that there have not been

2 consistent findings on the relationship between tissue heterogeneity and fracture status.

3 The effects of compositional changes on mechanical properties have been explored in several

4 studies. In one of these studies, elastic modulus determined from nanoindentation measurements

5 on biopsies from patients who have received long-term BP treatment demonstrated a reduction in

6 the heterogeneity of cortical bone elastic modulus compared to individuals with normal bone

7 [21]. At larger length scales, reference point indentation (RPI) measurements demonstrated a

8 deterioration in micro-indentation properties in AFF patients [3]. Furthermore, mechanical

9 testing on alendronate-treated canine cortical bone showed a decreasing trend in energy to

10 fracture [22] and reduced toughness [23]. In addition, a reduction in fatigue life using a

11 metabolic acidosis sheep model of osteoporosis and high dose alendronate-treated canine cortical

12 bone was observed compared to non-treated bone [24, 25].

13 In addition to experimental studies, computational modeling was also utilized to evaluate the

14 influence of compositional heterogeneity on mechanical properties. Several studies compared the

15 amount of energy dissipation in models that incorporate homogeneous and heterogeneous

16 material properties and concluded that the energy dissipation was higher in models with

17 heterogeneous material properties [26]. Tissue heterogeneity was found to play a more

18 significant role in inelastic properties than in elastic properties [27]. In addition, fracture

19 mechanics-based simulations demonstrated that the variation in material properties between

20 different compartments of cortical bone microstructure led to enhanced crack deflection

21 improving fracture toughness [28]. These findings indicate that a more uniform bone

22 composition both at submicro- and microscale may reduce the effectiveness of the fracture 
1 resistance of bone and may result in the progression of benign microcracks causing full fracture

$2[8,29]$.

3 In summary, the existing literature demonstrates the statistical correlation between

4 compositional changes and mechanical properties, but it does not directly identify the

5 mechanistic link between the material level changes and fracture behavior of bone. Particularly,

6 the direct effect of compositional changes on crack formation and propagation has not been

7 explicitly evaluated experimentally or computationally. In order to address this question, the goal

8 of the current study is to quantify the contribution of compositional heterogeneity on crack

9 growth in cortical bone using finite element modeling. The computational evaluation approach

10 developed here makes it possible to eliminate the confounding factors that exist in experimental

11 evaluations and to identify the direct influence of heterogeneity on crack growth behavior in

12 cortical bone. It presents direct evidence on how heterogeneity influences the fracture resistance

13 of cortical bone and may provide additional insight into how the prolonged BP use impacts the

14 mechanical properties of cortical bone. This may help improve the understanding of the possible 15 association of BPs with AFF.

\section{2. MATERIALS AND METHODS}

18 The influence of compositional heterogeneity on crack growth behavior in cortical bone was

19 assessed by simulating compact tension (CT) specimen fracture toughness tests on human

20 cortical bone with different material heterogeneity levels. The details of the modeling approach

21 are described in the subsequent sections. 


\section{$1 \quad 2.1$ Finite Element Model Description}

2 This study utilized transverse microscopy images of cortical bone from the mid-diaphysis of

3 tibiae of seven male donors with an age range of 39-85 years (average $=64.57 \pm 16.76$ years)

4 (Fig. 1a, 1c) denoted as M1 to M7 representing different microstructural distributions. The

5 microscopy images were obtained using a camera attached to a microscope under bright light at

$610 \mathrm{x}$ magnification and represent an area of $1.17 \times 0.89 \mathrm{~mm}^{2}$.

7 The microscopy images were converted to two-dimensional sketches by identifying the 8 microstructural features and approximating osteons and Haversian canals by ellipses (Fig. 2a, 2b)

9 following the procedure developed in [28]. This approximation was introduced to eliminate the

10 roughness of the osteon boundaries that can create numerical artefacts in the finite element

11 simulations. Following the generation of 2D sketches, 3D models were obtained by extruding the

12 models in the third dimension by an amount of $1 \mathrm{~mm}$ resulting in microstructural sections with

13 an approximate volume of $1 \mathrm{~mm}^{3}$ (Fig. 2c). The extrusion step relies on the assumption that the

14 entire bone volume has identical microstructure. Although this is generally not the case, due to

15 the small volume considered in the models, this assumption represents a valid approximation.

16 The detailed microstructural sections explicitly represent the interstitial bone, osteons, Haversian

17 canals, and cement lines (Fig. 1b, 1d). All models were generated using the finite element

18 software, ABAQUS (version 6.13, Simulia, Providence, RI). The detailed microstructural

19 sections were then inserted into a compact tension (CT) test specimen with a $1 \mathrm{~mm}^{3}$ cutout

20 section such that osteons were oriented perpendicular to the crack growth direction (Fig. 2d). The

21 dimensions of the $\mathrm{CT}$ specimen were based on previous experimental studies [30]. An initial

22 crack was introduced into the model in the mid-plane of the microstructural section with a depth

23 of $0.1 \mathrm{~mm}$. An incremental displacement boundary condition was applied on both top and bottom 
1 loading pin holes of the CT specimen to generate opening mode (Mode I) loading conditions.

2 This loading provides a stress state that leads to an incremental crack growth and prevents the

3 propagation of the crack through the entire volume of the specimen at once. The incremental

4 displacement was applied until the crack reached the homogeneous CT specimen section. Since

5 all models were evaluated at the same load level, the crack did not reach the end of the

6 microstructural section for all models. CT specimen was fixed in all directions at the mid-plane

7 at the far end from the loading point. All compartments of the finite element models were

8 meshed with tetrahedral elements with the exception of cement lines which were represented

9 with zero thickness triangular interface elements. The total number of elements in the models

10 ranged between 1.5 to 1.7 million. The size of the elements was $20 \mu \mathrm{m}$ in the microstructural

11 section.

12 The CT specimen and each compartment in the detailed microstructural section were

13 assigned isotropic elastic properties (Table 1). Crack formation and propagation in the osteons,

14 interstitial bone and CT specimen were modeled by cohesive extended finite element method

15 (XFEM) and in cement lines by cohesive interface elements as outlined in more detail in Section

16 2.2. In this modeling technique, ultimate strength and critical energy release rate need to be

17 specified in addition to the elastic properties of the bone. CT specimen section does not include

18 any microstructural detail, therefore, was assigned equivalent homogenized material properties

19 that were held constant for all simulations based on experimental data reported in the literature

20 [31-33] (Table 1). On the other hand, the material properties of the microstructural sections were

21 varied between simulations to represent the influence of material heterogeneity. A total of four

22 different cases was investigated for each model, including one simulation with homogeneous

23 material properties (HM) and three simulations with heterogeneous material properties. In the 
1 homogeneous models, each microstructural component was assigned uniform average material

2 properties based on the values reported in the literature [31, 34-36] (Table 1). The Poisson's

3 ratio was assigned a value of 0.3 and was kept constant for all models. In this study,

4 heterogeneity was represented by both the number of different material property values used and

5 spatial distribution (i.e. interstitial bone, osteons, and cement lines have their unique properties)

6 of these values in the models. In order to simulate different levels of heterogeneity, 5 (HT5), 10

7 (HT10), and 20 (HT20) material property sets composed of different elastic modulus, strength

8 and fracture toughness values were randomly generated using the average material properties and

9 their standard deviations for each microstructural compartment [31, 34-36] assuming a normal

10 distribution (Table 1). The details of the material properties used for M6 model with 10

11 heterogeneous material sets (HT10) is provided in supplemental materials section (Table S1-S3)

12 as a representative case. The difference between the average of the heterogeneous and

13 homogeneous material properties ranged predominantly between 0.1 to $15 \%$. Following this step,

14 these material properties were randomly assigned to the elements in the microstructural

15 compartments (Fig. 3). Each material property had equal representation among the elements

16 because of lack of experimental data on the possible distribution of these material properties. In

17 order to assess the influence of further variability in compositional heterogeneity simulations

18 with 100 different material property groups (HT100) were run for a subset of the models (M2

19 and M3). A MATLAB (MathWorks, Natick, MA) script was developed for the assignment of

20 heterogeneous material properties. In experimental studies reviewed in the Introduction, the

21 reduction in compositional heterogeneity was represented by a narrower distribution of

22 compositional variables. In order to demonstrate the similarity of the heterogeneity assignment

23 used in this study and the experimentally observed variations, two additional simulations for 
1 HT20 were run on M3 model by reducing and increasing the standard deviation of all material

2 properties by $20 \%$ based on the distribution widths for mineral to matrix ratio provided in the

3 literature [10]. These models evaluate the effect of narrower and wider distributions of material

4 properties for the same number of material sets.

5 In order to assess the influence of microstructure on the fracture behavior of cortical bone,

6 the microstructural parameters including percent osteonal area (On.Ar), osteon density (On.Dn),

7 percent porosity (Po), average area of a single osteon (s.On.Ar) and pore (S.Po.Ar) and cement

8 line density $(\mathrm{Cm} . \mathrm{Ln} . \mathrm{Dn}=\mathrm{Cement}$ line length/total area of bone $)$ were evaluated for each model

9 (Table 2) using the definitions identified previously [37, 38]. In addition, in order to assess the

10 influence of the osteon distribution on crack growth the moment of inertia of the osteons with

11 respect to their centroidal horizontal $\left(\mathrm{I}_{\mathrm{xc}}\right)$ and vertical axes $\left(\mathrm{I}_{\mathrm{yc}}\right)$ were calculated (Table 2$)$. The

12 area moment of inertia reflects how the osteons are distributed with respect to a chosen axis. A

13 smaller value of the moment of inertia indicates clustering of osteons around the centroidal axis,

14 whereas a larger value is indicative of a more even distribution of osteons.

\section{$15 \quad 2.2$ Cohesive Finite Element Modeling}

16 The crack initiation and propagation in the bone specimens were simulated by cohesive finite

17 element modeling that captures the nonlinear behavior of the process zone around a crack.

18 Cohesive modeling approach was incorporated in the models using two different formulations

19 including extended finite elements (XFEM) [39] in the osteons and interstitial bone and cohesive

20 interface elements [40] at the cement lines. Both formulations are represented by a traction-crack

21 opening displacement relationship that captures the physical processes occurring in the vicinity

22 of a propagating crack isolating the fracture process from the surrounding continuum constitutive

23 model. In the current study, a cohesive traction-displacement relationship that was established 
1 for bone in previous studies was employed [28, 41, 42] (Fig. 4). This relationship is based on an

2 effective traction-displacement concept which is a coupled representation of the normal and

3 shear behavior. Three material parameters define the cohesive model, including the ultimate

4 strength $\left(\sigma_{\mathrm{c}}\right)$, the critical energy release rate $\left(G_{\mathrm{c}}\right)$, and the critical crack opening displacement

$5\left(\delta_{\mathrm{u}}\right)$. Two of these three material properties are independent and are specified in the finite

6 element models based on experimental measurements reported in the literate as outlined in

7 Section 2.1. In this model, the damage initiation occurs through a quadratic interaction equation,

$8\left(T_{\mathrm{n}} / \sigma_{\mathrm{nc}}+T_{\mathrm{s}} / \sigma_{\mathrm{sc}}\right)^{2}=1$, where $T_{n}$, and $T_{s}$ are the current stress values in normal and shear directions

9 and $\sigma_{n c}$ and $\sigma_{s c}$ are critical normal and shear strengths. In the models, the shear behavior in both

10 directions is assumed to be the same [43]. Once the summation of these ratios equal to one,

11 damage starts to accumulate following the traction-displacement profile and full fracture occurs

12 when the current value of the energy release rate equals the critical energy release rate. This

13 corresponds to the point when there is no traction transferred between the crack surfaces. In the

14 interface element implementation of the cohesive model, the undamaged response of the

15 elements is represented by an initial ascending slope that is a penalty stiffness in the numerical

16 formulation (Fig. 4). On the other hand, for the XFEM formulation the initial ascending curve is

17 not required in the cohesive model as the crack domain is composed of solid elements which

18 have specified elastic behavior in the undamaged state.

19 The cohesive model was implemented as zero thickness interface elements which are

20 compatible with solid elements to represent the fracture behavior of cement lines. The crack

21 growth in the osteons, interstitial bone and CT specimen was formulated by XFEM. XFEM is

22 based on the partition of unity concept and incorporates local enrichment functions to represent

23 discontinuities created within the elements by the formation of cracks [39]. In this approach, the 
1 crack does not have to align with the element boundaries and can form at any region in an

2 element, therefore, is independent of the mesh. Specification of a crack domain that is composed

3 of general purpose solid finite elements is sufficient to activate the enrichment functions to

4 simulate crack initiation and propagation in the selected region. Therefore, in this approach a

5 predefined crack path does not have to be specified and the crack can initiate and propagate

6 anywhere in the crack domain.

\section{$7 \quad 2.3$ Analysis of Results}

8 The simulation results were assessed to compare the fracture behavior under different levels

9 of tissue heterogeneity and microstructure. A Python script was written using the ABAQUS

10 scripting interface to extract the simulation results. The fracture behavior was evaluated by

11 quantifying the amount of elements that have experienced a complete crack (denoted as "crack

12 volume") and that have accumulated damage but has not formed a full crack (denoted as

13 "damage volume"). In addition, the crack growth path was extracted to compare the influence of

14 tissue heterogeneity and microstructure on fracture resistance. The crack path was defined by the

15 elements that have undergone full crack formation. Uncracked regions were identified either by

16 elements that are damaged but have not formed a complete crack or by elements that have no 17 damage.

18 In order to evaluate the influence of microstructure on crack growth, the linear coefficients of

19 determination $\left(R^{2}\right)$ and statistical significance of the correlations $(p<0.05)$ were calculated

20 between the microstructural parameters outlined in Section 2.1 (On.Ar, On.Dn, Po, s.On.Ar,

21 s.Po.Ar, Cm.Ln.Dn, $\left.\mathrm{I}_{\mathrm{xc}}, \mathrm{I}_{\mathrm{yc}}\right)$ and the crack and damage volume. The correlations were reported

22 for the models with homogeneous material properties since the same trends were obtained for

23 heterogeneous material property sets. All statistical analyses were performed using MATLAB. 


\section{3. RESULTS}

2 Finite element simulations were performed with both homogeneous and heterogeneous 3 material properties using all the cortical bone models. The simulation results predicted that the

4 crack volume was the highest for the homogeneous material properties for all models (Fig. 5).

5 The lowest crack volume was attained in the HT10 models with the exception of M6 (Fig. 5).

6 The reduction in crack volume with respect to the homogeneous material properties ranged 7 between $1.9 \%$ - $41 \%$ in HT5 and $18.3 \%$ - 45.5\% in HT10 models. Further increasing the 8 heterogeneity from HT10 did not provide additional beneficial effects and in all cases with the 9 exception of M6 resulted in a higher crack volume than the HT10 case (Fig. 5). The crack 10 volume reduction in these models ranged between $0 \%-31 \%$ in HT20 and $13 \%-16.4 \%$ in 11 HT100 models compared to the homogeneous cases which was less than the HT10 models. (Fig. $125)$.

13 The evaluation of damage volume in the models showed that homogeneous models 14 predominantly had lower damage than heterogeneous models (Fig. 6) while they had the highest 15 crack volume. In most models, the highest damage formation occurred in HT10 models which 16 had the lowest crack volume (Fig. 6). However, there was not a consistent trend among all 17 heterogeneous models.

18 The evaluation of the crack propagation path confirmed that there was extensive crack

19 formation with limited areas of damage in the homogeneous models particularly in the interstitial 20 bone (Fig. 7). The heterogeneity in the material properties led to a more variable distribution of 21 damage in all models compared to the homogeneous material properties (Fig. 7). This resulted in

22 uncracked sections of bone with low damage accumulation during crack propagation. Most of 23 the crack formation occurred in the interstitial bone. There was occasional full penetration of 
1 cracks into osteons but in most cases there was either no damage or low damage accumulation in

2 parts of the osteons particularly in heterogeneous models (Fig. 7). The number of elements that

3 attained full crack formation at the cement lines was very small in homogeneous models

4 compared to the heterogeneous models (Fig. 8).

5 Although, the crack growth trends with heterogeneity were the same in all models, there was

6 a substantial difference in the amount of crack formation between the models (Fig. 5 and 7). This

7 difference stemmed from the variation in the microstructure between the samples (Table 2).

8 Statistically significant correlations were observed between $\mathrm{I}_{\mathrm{xc}}$, On.Ar and crack volume (Table

9 3). The crack volume decreased with increasing On.Ar and $I_{x c}$. When $I_{x c}$ was normalized with

10 On.Ar in each model, there was no statistically significant relationship with the crack volume.

11 Damage volume did not demonstrate any statistically significant relationships with

12 microstructural parameters. Furthermore, the lowest reduction in the crack volume with

13 heterogeneity occurred in the specimen with the highest porosity.

14 The comparison of the heterogeneity used in the current study to the narrower and wider

15 distributions of material property using the same material property sets showed that narrower

16 distribution of material properties led to a slightly higher crack volume and wider distribution

17 resulted in a lower crack volume compared to the homogeneous case. Furthermore, comparison

18 of the results to the heterogeneous model with the original standard deviation values from the

19 literature showed that narrower and wider distribution of material properties resulted in higher

20 and a slightly lower crack volume compared to the original heterogeneous model, respectively.

21 (Fig. 9). 


\section{4. DISCUSSION}

2 This study presented a new computational approach to evaluate the influence of reduced

3 material heterogeneity on crack propagation and fracture resistance of cortical bone. The novelty

4 of the computational approach in this study comes from the incorporation of XFEM and cohesive

5 interface elements in three-dimensional models of cortical bone crack growth at the microscale

6 which was not undertaken previously. In addition, earlier studies have not incorporated complete

7 spatial material heterogeneity in the models at the microstructural level in cortical bone. The

8 simulation results provide unique information on the extent of crack growth under different

9 material heterogeneity levels and directly capture the effect of changes in the material

10 heterogeneity on crack propagation in cortical bone by eliminating other confounding factors that

11 may exist in experimental studies.

12 The simulation results predicted that reduced heterogeneity adversely affects the fracture

13 resistance in cortical bone and may result in progression of microcracks into full fracture as

14 previously hypothesized in the literature [8]. The results also demonstrated that there is a non-

15 linear relationship between heterogeneity and fracture resistance. This is highlighted by an

16 increase in fracture resistance up to certain level of heterogeneity which declined with a further

17 increase in heterogeneity. The negative effects of reduced heterogeneity found in this study are

18 in line with experimental studies that related the deterioration of mechanical properties to a

19 reduction in the heterogeneity of material properties [21]. Previous studies established a

20 statistical correlation between reduced heterogeneity and BP use and fractures [9-15]. The

21 results of the current study combined with these observations in the literature indicate that severe

22 suppression of bone turnover may have adverse effects on fracture resistance in cortical bone as

23 it will significantly reduce the compositional heterogeneity. In the current study, there was no 
1 additional improvement in fracture resistance of cortical bone with further increase in

2 heterogeneity after a certain level. The results demonstrated that the lowest crack volume was

3 attained in the HT10 models with the exception of M6 model. Increasing the heterogeneity

4 further predominantly resulted in higher crack volume amount than HT10 but lower or equal

5 crack volume compared to homogeneous models. Although the trends were similar, the

6 percentage differences in crack volume varied between different heterogeneity levels among

7 models. This may indicate an interaction between the microstructure and the material

8 heterogeneity. The overall results from the simulations demonstrate that benefits from increasing

9 heterogeneity do not improve after a certain level. This may suggest the existence of an optimum

10 tissue heterogeneity that maximizes the crack growth resistance in cortical bone. In addition, this

11 observation may reconcile the conflicting findings of higher mineralization heterogeneity in 12 certain fracture cohorts $[18,19]$.

13 Homogeneous models demonstrated limited areas of damage with extensive crack formation,

14 whereas heterogeneous models showed larger areas of damage with smaller amount of full crack

15 formation. These results may suggest a more effective energy dissipation mechanism due to

16 heterogeneity in the material properties. The variation in the amount of damage accumulation

17 observed in the heterogeneous models resulted in uncracked sections of bone with low damage

18 that bridge the crack. This mechanism contributed to the increased fracture resistance in the

19 heterogeneous models by providing more resistance against crack propagation. These

20 observations support the suggested link between reduction in compositional heterogeneity and

21 AFFs. AFFs are insufficiency fractures that occur as a result of non-traumatic events such as a

22 fall from a standing height and present a transverse configuration [1]. The transverse fracture line

23 associated with AFFs has been suggested as evidence of unimpeded crack growth and failure of 
1 fracture toughening mechanisms that normally exist in bone [8]. More effective energy

2 dissipation and bridging that is observed in the heterogeneous models in the current study

3 indicate that reduction in material heterogeneity may adversely affect the fracture response of

4 cortical bone and may lead to extensive crack propagation without resistance.

5 The results also showed that the cortical bone microstructure, particularly the distribution of

6 osteons and osteonal area, play a significant role in determining the extent of crack growth. In

7 the simulations, the reduction in osteonal area led to a higher amount of crack growth. The same

8 trend was observed in experimental studies that showed a decreasing trend in fracture toughness

9 [44] and energy to fracture [32] with decreasing osteonal area in human cortical bone. The

10 simulations also showed that the reduction in the moment of inertia with respect to the centroidal

11 axis parallel to the crack growth direction $\left(\mathrm{I}_{\mathrm{xc}}\right)$ led to a larger extent of crack growth. The area

12 moment of inertia reflects how the osteons are distributed with respect to a chosen axis. In the

13 current study, the osteonal moment of inertia was calculated with respect to the two main axes of

14 the model i.e. $\mathrm{x}$-axis $\left(\mathrm{I}_{\mathrm{xc}}\right)$ and $\mathrm{y}$-axis $\left(\mathrm{I}_{\mathrm{yc}}\right)$ which are parallel and perpendicular to the crack

15 growth direction, respectively. $\mathrm{I}_{\mathrm{xc}}$ represents how the osteons are distributed across the width of

16 the crack plane and provides a measure of the barrier effect that the osteons have on crack

17 extension. A smaller value of $\mathrm{I}_{\mathrm{xc}}$ indicates clustering of osteons around the centroidal axis,

18 whereas a larger value is indicative of a more even distribution of osteons across the width of the

19 bone sample. The correlation results in this study showed that evenly distributed osteons can be

20 more effective in preventing crack propagation by creating a barrier compared to clustered

21 osteons which would leave large areas of interstitial bone without any effective mechanism to

22 stop and deflect a growing crack. On the other hand, no correlation was observed between $\mathrm{I}_{\mathrm{yc}}$ and

23 the crack volume. $I_{y c}$ captures how far the osteons are located horizontally from the center of the 
1 crack plane and does not directly provide information on how the osteons are distributed with

2 respect to the crack growth direction. As a result, $\mathrm{I}_{\mathrm{yc}}$ is not correlated with the crack volume. In

3 addition, if the crack front encounters osteons earlier on the crack plane the crack growth is

4 impeded significantly irrespective of how the osteons are distributed with respect to the $\mathrm{x}$-axis.

5 However, $\mathrm{I}_{\mathrm{yc}}$ does not directly reflect the location of the initial group of osteons with respect to

6 the crack front as $\mathrm{I}_{\mathrm{yc}}$ is calculated based on how all osteons, not just the first row of osteons, are

7 distributed with respect to the $y$-axis on the crack plane.

8 The crack growth process in the simulations is in line with the observations from

9 experimental fracture toughness tests performed on cortical bone in the transverse orientation

10 [35, 45]. Simulations demonstrated that the cracks initially formed and grew in the interstitial

11 bone and were deflected at cement lines leading to crack deflection and twisting around osteons

12 which was similar to experimentally observed crack growth behavior. The penetration of cracks

13 into osteons was mostly observed in homogeneous models. The cracks were diverted by the

14 cement lines, particularly in heterogeneous models, capturing the experimentally observed crack

15 deflection behavior by cement lines $[35,45]$.

16 The strength of the current study is that it successfully isolated the changes in the material

17 heterogeneity from other factors and identified its individual and direct effect on fracture

18 resistance of cortical bone. The use of models based on different human cortical bone samples

19 made it possible to confirm that the findings on the effect of heterogeneity is independent of the

20 microstructure of cortical bone. This was confirmed by the same trends observed in the reduction

21 of crack volume in heterogeneous models compared to the homogeneous models for all

22 microstructures (Fig. 5). 
In the current study, we utilized crack volume to represent the fracture resistance in the

2 models instead of a macro-crack length due to the crack deflection occurring in three-dimensions

3 that influences fracture resistance. The crack volume shows how much crack is forming in each

4 model at a given load. At the same load level, if there is more crack formation in a model this

5 indicates that it is easier to form cracks in this model and the fracture resistance is lower in this

6 model compared to a model which has less crack formation. The crack volume also captures the

7 difference in the entire crack formation process between models as can be seen in Figure 7. In

8 addition, the majority of the cracked elements in all models are connected to the main crack and

9 contribute to the main crack propagation. As a result, the crack volume represents the level of

10 fracture resistance in the models.

11 In this study, heterogeneity was represented by both the number of different material 12 property sets used and spatial distribution of these values in the models. In experimental studies,

13 the reduction in compositional heterogeneity was represented by a narrower distribution of the

14 compositional variables. Narrower and wider material distributions represent a smaller and larger

15 number of distinct material property sets in the bone, respectively. There is no quantitative

16 information in the literature on the change in the standard deviation of material properties as a

17 result of these compositional changes. Therefore, in order to capture the change in compositional

18 heterogeneity distribution in the simulations, the narrower and wider distributions were

19 simulated with varying the number of distinct material property sets. In order to demonstrate the

20 similarity of these approaches, two simulations using 20 heterogeneous material sets were run on

21 M3 model by reducing and increasing the standard deviation values for all material properties

22 which demonstrated similar trends as shown in our implementation of heterogeneity. 
One of the limitations of the current study is the use of material distribution based on

2 experimentally measured average material properties and their standard deviations instead of

3 locally measured bone material properties specifically for each bone sample. This approach was

4 chosen due to the lack of specimen specific experimental data in the literature. However, the 5 established new computational modeling approach can readily be combined with direct 6 experimental local measurements of tissue compositional and material properties on bone 7 biopsies to provide valuable insight into patient-specific fracture resistance of cortical bone. 8 Another limitation is obtaining a 3D image of the bone sections by extruding the 2D images. The 9 extrusion step relies on the assumption that the entire bone volume has identical microstructure. 10 Although this is generally not the case, due to the small volume considered in the models, and 11 the extrusion amount being only $1 \mathrm{~mm}$, this assumption is not expected to lead to a significant 12 influence on the results. In the current study, only material heterogeneity was altered in the 13 models and material property changes due to tissue age or remodeling were not incorporated. In 14 order to capture the full effect of suppressed bone turnover on the fracture resistance of cortical 15 bone other factors that influence the material properties need to be incorporated in the models as 16 well. Previous studies have shown that BP use increases advanced glycation end products 17 (AGEs) [46] which is known to reduce the fracture toughness of cortical bone [47, 48] and 18 increases mineralization [9] elevating brittleness. The effect of these changes can be incorporated 19 in the cohesive models by replacing the material properties listed in Table 1 for healthy bone 20 with properties measured on bone that has undergone BP treatment. However, a complete set of 21 experimental data for BP treated bone that reflect these changes is not currently available in the

22 literature. As a result, it was not possible to implement these changes in the current models.

23 Furthermore, the focus of this study was to evaluate the individual influence of heterogeneity on 
1 fracture resistance in the absence of other effects. Although these changes were not included in

2 the current study, the computational approach presented here have the capability to incorporate

3 these factors and can be used to further evaluate the full range of effects of BP use on the

4 fracture resistance of cortical bone. An additional limitation of this study is that although the

5 bone specimens were obtained from individuals with no known metabolic bone diseases, there is

6 not sufficient information in the donor registries about osteoporosis or anti-resorptive treatment

7 state. As a result, the bone specimens may not be representative of cortical bone microstructure

8 under BP treatment. However, the bone specimens chosen cover a wide range of osteonal area

9 and density and the trends obtained for the heterogeneous models are independent of the

10 microstructure. As a result, this issue is not expected to influence the findings in this study.

11 In summary, this study developed a new computational modeling approach that evaluates the

12 influence of compositional heterogeneity on fracture resistance of cortical bone. The simulation

13 results establish new information about the adverse effects of reduced heterogeneity on crack

14 growth in cortical bone. This new computational modeling approach provides a tool that can be

15 used to establish the mechanistic link between material level changes and fracture behavior of

16 cortical bone. It also can help improve the understanding of the effects of material level changes

17 due to prolonged BP use on the overall bone fracture behavior. It may also bring additional

18 insight into the causes of unusual fractures such as AFF and their possible association with long

19 term BP use.

\section{ACKNOWLEDGEMENTS}

22 This study is funded by National Science Foundation Award Number CMMI- 1434412. 
Table 1: Average and standard deviation of elastic and cohesive model properties used in the simulations based on the experimental data reported in the literature. Note that $E$ is the elastic modulus, $\sigma_{n c}, \sigma_{s c}$, are the normal and shear strength, respectively, $K_{\text {eff }}$ is the effective stress intensity factor, and $G_{c}$ is the effective critical energy release rate.

\begin{tabular}{|c|c|c|c|c|}
\hline & Osteons & Interstitial Bone & Cement Lines & CT Specimen \\
\hline $\boldsymbol{E}(\mathrm{GPa})$ & $22.5 \pm 13^{1}$ & $25.8 \pm 0.7^{1}$ & N/A & $23.6^{1}$ \\
\hline$\sigma_{\text {nc }}(\mathrm{MPa})$ & $53 \pm 10.7^{2}$ & $53 \pm 10.7^{2}$ & $53 \pm 10.7^{2}$ & $103.76^{3}$ \\
\hline$\sigma_{\mathrm{sc}}(\mathrm{MPa})$ & $68 \pm 3.4^{2}$ & $68 \pm 3.4^{2}$ & $7.96 \pm 1.99^{4}$ & $68^{2}$ \\
\hline $\boldsymbol{K}_{\text {eff }}\left(\mathrm{MPa} \mathrm{m}^{1 / 2}\right)$ & $1 \pm 0.2^{5}$ & $1 \pm 0.2^{5}$ & $1 \pm 0.2^{5}$ & N/A \\
\hline$\overline{\boldsymbol{G}_{\mathbf{c}}\left(\mathrm{kJ} / \mathrm{m}^{2}\right)}$ & $K_{\mathrm{eff}}^{2} / \mathrm{E}^{7}$ & $K_{\mathrm{eff}}^{2} / \mathrm{E}^{7}$ & $K_{\text {eff }}^{2} / \mathrm{E}^{7}$ & $1.16^{6}$ \\
\hline
\end{tabular}

\footnotetext{
${ }^{1}$ From reference [31] based on measurements on tibia from donors with an age range of 57-61

${ }^{2}$ From reference [36] based on measurements on femur from donors with an age range of 23-63

${ }^{3}$ From reference [32] based on measurements on femur from donors with an age range of 20-102

${ }^{4}$ From reference [34] based on measurements on humerus from donors with an age range of 47-57

${ }^{5}$ From reference [35] based on measurements on humerus from donors with an age range of 37-41

${ }^{6}$ From reference [33] based on measurements on femur from donors with an age range of 35-92

${ }^{7} G_{\mathrm{c}}$ is calculated based on $K_{\text {eff }}$ using an elastic modulus of $20 \mathrm{GPa}$ [35].
}

Table 2: Summary of microstructural parameters for each cortical bone model.

\begin{tabular}{l|cccccccc}
\hline & M1 & M2 & M3 & M4 & M5 & M6 & M7 & Average \\
\hline On.Ar $(\%)$ & 60.4 & 64.6 & 44.6 & 53.5 & 67.7 & 45.6 & 72.8 & $58.5 \pm 10.9$ \\
\hline On.Dn $\left(\mathbf{m m}^{-2}\right)$ & 11.6 & 20.3 & 20.3 & 15.5 & 13.5 & 20.3 & 19.3 & $17.3 \pm 3.7$ \\
\hline Po $(\%)$ & 4.93 & 5.02 & 3.01 & 4.46 & 4.40 & 4.31 & 10.15 & $5.2 \pm 2.3$ \\
\hline s.On.Ar $\left(\mathbf{m m}^{2}\right) \mathbf{x 1 0}$ & 48 & 28 & 20 & 32 & 47 & 20 & 32 & $32.4 \pm 11.4$ \\
\hline s.Po.Ar $\left(\mathbf{m m}^{2}\right) \mathbf{x 1 0}$ & 4 & 3 & 2 & 3 & 3 & 2 & 8 & $3.6 \pm 2.1$ \\
\hline Cm.Ln.Dn $\left(\mathbf{m m} \mathbf{m m m}^{-3}\right)$ & 7.08 & 8.82 & 8.44 & 7.72 & 6.84 & 9.09 & 5.77 & $7.7 \pm 1.2$ \\
\hline $\mathbf{I}_{\mathbf{x c}}\left(\mathbf{m m}^{4}\right) \mathbf{x 1 0 ^ { - 3 }}$ & 58.0 & 67.6 & 41.8 & 50.8 & 70.8 & 49.2 & 69.8 & $58.3 \pm 11.4$ \\
\hline $\mathbf{I}_{\mathbf{y c}}\left(\mathbf{m m}^{4}\right) \mathbf{x 1 0 ^ { - 3 }}$ & 38.3 & 25.9 & 18.3 & 30.8 & 30.5 & 27.6 & 42.4 & $30.5 \pm 8$ \\
\hline
\end{tabular}


Table 3: Correlations between microstructural parameters and crack volume. The first row corresponding to each parameter is the coefficient of determination $\left(R^{2}\right)$ and the second row shown in italic is the statistical significance value $(p)$. Significant correlations $(p<0.05)$ are shown in bold. The regression equations corresponding to the statistically significant relationships are shown in the footnote.

\begin{tabular}{l|cccccccc}
\hline & On.Ar & On.Dn & S.On.Ar & Po & S.Po.Ar & I $_{\mathbf{x c}}$ & $\mathbf{I}_{\mathbf{y c}}$ & Cm.Ln.Dn \\
\hline Crack & $\mathbf{0 . 7 1}$ & 0.15 & 0 & 0.41 & 0.39 & $\mathbf{0 . 7 9}^{1}$ & 0.26 & 0.19 \\
Volume & 0.017 & 0.39 & 0.96 & 0.12 & 0.13 & 0.007 & 0.24 & 0.32 \\
\hline
\end{tabular}

${ }^{\mathrm{I}}$ Crack Volume $=-0.3365 \mathrm{I}_{\mathrm{xc}}+0.0290$

${ }^{2}$ Crack Volume $=-0.0342 \mathrm{On} . \mathrm{Ar}+0.0293$ 


\section{FIGURE CAPTIONS}

Figure1: (a, c) Transverse microscopy images of human cortical bone from the mid-diaphysis of tibiae of male donors in order of M1 to M7 (b, d) 3D finite element models of microscopy images.

Figure 2: (a) A sample transverse microscopy image of cortical bone (M5) with outline of the cement lines (b) 2D detailed sketch based on the image in (a). (c) 3D finite element model obtained by extruding the 2D sketch in (b). (d) Finite element model of the CT specimen showing the location (marked by the circle) where the detailed microstructure cell was placed.

Figure 3: A sample heterogeneous material distribution for M3 showing (a) 5 (HT5), (b) 10 (HT10), and (c) 20 (HT20) material sets. Note that each color in the pictures corresponds to a different material set.

Figure 4: Traction $(T)$ - crack opening $(\delta)$ displacement relationship defining the cohesive model where $\sigma_{c}$ is the critical strength, $\delta_{u}$ is the ultimate crack opening displacement, and $G_{\mathrm{c}}$ is the critical energy release rate that corresponds to fracture energy. Note that the initial ascending line shown in dotted lines is used only in the interface element formulation and is not required for the XFEM formulation.

Figure 5: Comparison of crack volume between different levels of heterogeneity for all specimens. The solid colors and bordered white regions indicate the normalized crack volume in interstitial bone and osteons, respectively. Note that all crack volume values were normalized by the highest crack volume in all models to demonstrate the percent difference between different heterogeneity levels and models.

Figure 6: Comparison of damage volume between different levels of heterogeneity for all specimens. The solid colors and bordered white regions indicate the normalized damage volume in interstitial bone and osteons, respectively. Note that all damage volume values were 
normalized by the highest damage volume in all models to demonstrate the percent difference between different heterogeneity levels and models.

Figure 7: Representative planar view of crack growth for models (a) M2, (b) M3 and (c) M6 with homogeneous and heterogeneous material distributions. The colors represent the damage accumulation in a rainbow contour where red is full crack and dark blue is the lowest damage. The regions shown by light and dark gray elements correspond to no damage accumulation. The dashed arrows represent the crack growth direction.

Figure 8: Representative 3D view of (a) damaged and cracked elements and (b) only cracked elements including cement lines for M3-HM model. The only cracked cement line element in the HM model is behind the cracked osteon elements and is not visible. Its location is marked with an arrow in the image. Representative 3D view of (c) damaged and cracked elements (d) only cracked elements including cement lines for M3-HT10 model. The regions with cracked cement line elements are shown with arrows. Note that in (a) and (c) the elements colored in green, blue, and red show undamaged, damaged, and cracked regions, respectively. In (b) and (d) blue represent the cracked elements in the cement lines and red corresponds to the cracked elements in interstitial and osteonal bone.

Figure 9: Comparison of normalized crack volume in M3 between HM and HT20 with original, narrower $(80 \%)$ and wider (120\%) standard deviation ranges for all material properties listed in Table 1. The solid colors and bordered white regions indicate the normalized crack volume in interstitial bone and osteons, respectively. 


\section{REFERENCES}

1 [1] Shane E, Burr D, Abrahamsen B, Adler RA, Brown TD, Cheung AM, Cosman F, Curtis JR, Dell R, Dempster DW, Ebeling PR, Einhorn TA, Genant HK, Geusens P, Klaushofer K, Lane JM, McKiernan F, McKinney R, Ng A, Nieves J, O'Keefe R, Papapoulos S, Howe TS, van der Meulen MCH, Weinstein RS, Whyte MP. Atypical subtrochanteric and diaphyseal femoral fractures: Second report of a task force of the American Society for Bone and Mineral Research. Journal of Bone and Mineral Research 2014;29: 1-24.

[2] Shane E, Burr D, Ebeling PR, Abrahamsen B, Adler RA, Brown TD, Cheung AM, Cosman F, Curtis JR, Dell R, Dempster D, Einhorn TA, Genant HK, Geusens P, Klaushofer K, Koval K, Lane JM, McKiernan F, McKinney R, Ng A, Nieves J, O'Keefe R, Papapoulos S, Sen HT, van der Meulen MCH, Weinstein RS, Whyte M. Atypical subtrochanteric and diaphyseal femoral fractures: Report of a task force of the american society for bone and mineral Research. Journal of Bone and Mineral Research 2010;25: 2267-2294.

[3] Güerri-Fernández RC, Nogués X, Quesada Gómez JM, Torres del Pliego E, Puig L, García-Giralt N, Yoskovitz G, Mellibovsky L, Hansma PK, Díez-Pérez A. Microindentation for in vivo measurement of bone tissue material properties in atypical femoral fracture patients and controls. Journal of Bone and Mineral Research 2013;28: 162-168.

[4] Gedmintas L, Solomon DH, Kim SC. Bisphosphonates and risk of subtrochanteric, femoral shaft, and atypical femur fracture: A systematic review and meta-analysis. Journal of Bone and Mineral Research 2013;28: 1729-1737.

[5] Dell RM, Adams AL, Greene DF, Funahashi TT, Silverman SL, Eisemon EO, Zhou H, Burchette RJ, Ott SM. Incidence of atypical nontraumatic diaphyseal fractures of the femur. Journal of Bone and Mineral Research 2012;27: 2544-2550.

[6] Meier RH, Perneger TV, Stern R, Rizzoli R, Peter RE. INcreasing occurrence of atypical femoral fractures associated with bisphosphonate use. Archives of Internal Medicine 2012;172: 930-936.

[7] Schilcher J, Koeppen V, Aspenberg P, Michaëlsson K. Risk of atypical femoral fracture during and after bisphosphonate use: Full report of a nationwide study. Acta Orthopaedica 2015;86: 100-107.

29 [8] Ettinger B, Burr DB, Ritchie RO. Proposed pathogenesis for atypical femoral fractures: lessons from materials research. Bone 2013;55: 495-500.

[9] Boskey AL, Spevak L, Weinstein RS. Spectroscopic markers of bone quality in alendronate-treated postmenopausal women. Osteoporosis International 2009;20: 793-800.

[10] Donnelly E, Meredith DS, Nguyen JT, Gladnick BP, Rebolledo BJ, Shaffer AD, Lorich DG, Lane JM, Boskey AL. Reduced cortical bone compositional heterogeneity with bisphosphonate treatment in postmenopausal women with intertrochanteric and subtrochanteric fractures. Journal of Bone and Mineral Research 2012;27: 672-678.

[11] Bala Y, Depalle B, Farlay D, Douillard T, Meille S, Follet H, Chapurlat R, Chevalier J, Boivin G. Bone micromechanical properties are compromised during long-term alendronate therapy independently of mineralization. Journal of Bone and Mineral Research 2012;27: 825834.

[12] Roschger P, Rinnerthaler S, Yates J, Rodan GA, Fratzl P, Klaushofer K. Alendronate increases degree and uniformity of mineralization in cancellous bone and decreases the porosity in cortical bone of osteoporotic women. Bone 2001;29: 185-191.

44 [13] Monier-Faugere M-C, Geng Z, Paschalis EP, Qi Q, Arnala I, Bauss F, Boskey AL, 45 Malluche $\mathrm{HH}$. Intermittent and continuous administration of the bisphosphonate ibandronate in 
ovariohysterectomized beagle dogs: effects on bone morphometry and mineral properties. Journal of Bone and Mineral Research 1999;14: 1768-1778.

[14] Boskey AL. Bone composition: relationship to bone fragility and antiosteoporotic drug effects. BoneKEy Reports 2013;2.

[15] Gourion-Arsiquaud S, Lukashova L, Power J, Loveridge N, Reeve J, Boskey AL. Fourier transform infrared imaging of femoral neck bone: Reduced heterogeneity of mineral-to-matrix and carbonate-to-phosphate and more variable crystallinity in treatment-naive fracture cases compared with fracture-free controls. Journal of Bone and Mineral Research 2013;28: 150-161.

[16] Ciarelli TE, Tjhia C, Rao DS, Qiu S, Parfitt AM, Fyhrie DP. Trabecular packet-level lamellar density patterns differ by fracture status and bone formation rate in white females. Bone 2009;45: 903-908. [17] Boskey AL, Donnelly E, Boskey E, Spevak L, Ma Y, Zhang W, Lappe J, Recker RR. Examining the relationships between bone tissue composition, compositional heterogeneity, and fragility fracture: A matched case-controlled FTIRI study. Journal of Bone and Mineral Research 2015; 10.1002/jbmr.2759.

[18] Tamminen IS, Misof BM, Roschger P, Mäyränpää MK, Turunen MJ, Isaksson H, Kröger $\mathrm{H}$, Mäkitie O, Klaushofer K. Increased heterogeneity of bone matrix mineralization in pediatric patients prone to fractures: a biopsy study. Journal of Bone and Mineral Research 2014;29: 1110-1117.

[19] Bousson V, Bergot C, Wu Y, Jolivet E, Zhou LQ, Laredo J-D. Greater tissue mineralization heterogeneity in femoral neck cortex from hip-fractured females than controls. A microradiographic study. Bone 2011;48: 1252-1259.

[20] Roschger P, Misof B, Paschalis E, Fratzl P, Klaushofer K. Changes in the degree of mineralization with osteoporosis and its treatment. Current osteoporosis reports 2014;12: 338350.

[21] Tjhia CK, Odvina CV, Rao DS, Stover SM, Wang X, Fyhrie DP. Mechanical property and tissue mineral density differences among severely suppressed bone turnover (SSBT) patients, osteoporotic patients, and normal subjects. Bone 2011;49: 1279-1289.

[22] Acevedo C, Bale H, Gludovatz B, Wat A, Tang SY, Wang M, Busse B, Zimmermann EA, Schaible E, Allen MR. Alendronate treatment alters bone tissues at multiple structural levels in healthy canine cortical bone. Bone 2015;81:352-363.

[23] Allen MR, Reinwald S, Burr DB. Alendronate reduces bone toughness of ribs without significantly increasing microdamage accumulation in dogs following 3 years of daily treatment. Calcified Tissue International 2008;82: 354-360.

[24] Brock GR, Chen JT, Ingraffea AR, MacLeay J, Pluhar GE, Boskey AL, van der Meulen MC. The effect of osteoporosis treatments on fatigue properties of cortical bone tissue. Bone Reports 2015;2: 8-13.

[25] Bajaj D, Geissler JR, Allen MR, Burr DB, Fritton J. The resistance of cortical bone tissue to failure under cyclic loading is reduced with alendronate. Bone 2014;64: 57-64.

40 [26] Tai K, Dao M, Suresh S, Palazoglu A, Ortiz C. Nanoscale heterogeneity promotes 41 energy dissipation in bone. Nature Materials 2007;6: 454-462.

42 [27] Yao H, Dao M, Carnelli D, Tai K, Ortiz C. Size-dependent heterogeneity benefits the 43 mechanical performance of bone. Journal of the Mechanics and Physics of Solids 2011;59: 644474. 
[28] Mischinski S, Ural A. Interaction of microstructure and microcrack growth in cortical bone: a finite element study. Computer Methods in Biomechanics and Biomedical Engineering 2013;16: 81-94.

[29] Ritchie RO. The conflicts between strength and toughness. Nature Materials 2011;10: 817-822.

[30] Vashishth D, Behiri JC, Bonfield W. Crack growth resistance in cortical bone: concept of microcrack toughening. J Biomech 1997;30: 763-9.

8 [31] Rho J-Y, Tsui TY, Pharr GM. Elastic properties of human cortical and trabecular lamellar 9 bone measured by nanoindentation. Biomaterials 1997;18: 1325-1330.

[32] McCalden RW, McGeough JA, Barker MB, Court-Brown CM. Age-related changes in the tensile properties of cortical bone. The relative importance of changes in porosity, mineralization, and microstructure. The Journal of Bone \& Joint Surgery American Volume 1993;75: 1193-205.

[33] Zioupos P, Currey JD. Changes in the stiffness, strength, and toughness of human cortical bone with age. Bone 1998;22: 57-66.

[34] Dong XN, Zhang X, Guo XE. Interfacial strength of cement lines in human cortical bone. MCB-Tech Science Press 2005;2: 63.

[35] Koester KJ, Ager J, Ritchie R. The true toughness of human cortical bone measured with realistically short cracks. Nature Materials 2008;7: 672-677.

[36] Reilly DT, Burstein AH. The elastic and ultimate properties of compact bone tissue. Journal of Biomechanics 1975;8: 393-405.

[37] Ural A, Vashishth D. Interactions between microstructural and geometrical adaptation in human cortical bone. Journal of Orthopaedic Research 2006;24: 1489-98.

[38] Gibson VA, Stover SM, Gibeling JC, Hazelwood SJ, Martin RB. Osteonal effects on elastic modulus and fatigue life in equine bone. Journal of Biomechanics 2006;39: 217-225.

[39] Moës N, Belytschko T. Extended finite element method for cohesive crack growth. Engineering Fracture Mechanics 2002;69: 813-833.

[40] Ortiz M, Pandolfi A. Finite-deformation irreversible cohesive elements for threedimensional crack-propagation analysis. International Journal for Numerical Methods in Engineering 1999;44: 1267-1282.

[41] Ural A. Prediction of Colles' fracture load in human radius using cohesive finite element modeling. Journal of Biomechanics 2009;42: 22-8.

[42] Ural A, Vashishth D. Cohesive finite element modeling of age-related toughness loss in human cortical bone. Journal of Biomechanics 2006;39: 2974-82.

[43] Camanho PP, Davila CG, De Moura MF. Numerical Simulation of Mixed-Mode Progressive Delamination in Composite Materials. Journal of Composite Materials 2003;37: 1415-1438.

[44] Yeni YN, Brown CU, Wang Z, Norman TL. The influence of bone morphology on fracture toughness of the human femur and tibia. Bone 1997;21: 453-9.

40 [45] Koester KJ, Barth HD, Ritchie RO. Effect of aging on the transverse toughness of human 41 cortical bone: Evaluation by R-curves. Journal of the Mechanical Behavior of Biomedical 42 Materials 2011;4: 1504-1513.

43 [46] Tang SY, Allen MR, Phipps R, Burr DB, Vashishth D. Changes in non-enzymatic 44 glycation and its association with altered mechanical properties following 1-year treatment with 45 risedronate or alendronate. Osteoporosis International 2009;20: 887-894. 
1 [47] Poundarik AA, Wu P-C, Evis Z, Sroga GE, Ural A, Rubin M, Vashishth D. A direct role 2 of collagen glycation in bone fracture. Journal of the Mechanical Behavior of Biomedical 3 Materials 2015;50: 82-92.

4 [48] Wang X, Bank RA, TeKoppele JM, Agrawal CM. The role of collagen in determining 5 bone mechanical properties. Journal of Orthopaedic Research 2001;19: 1021-6. 


\section{FigureV}

Figure 1

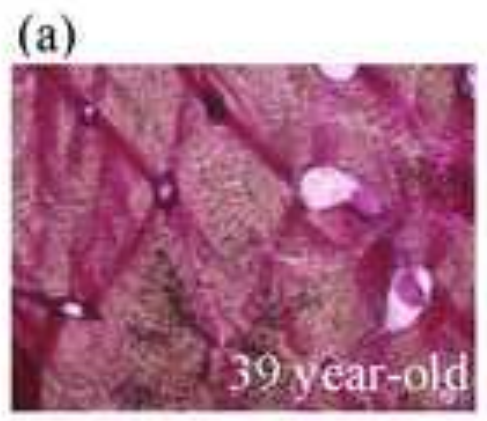

(b)

(c)
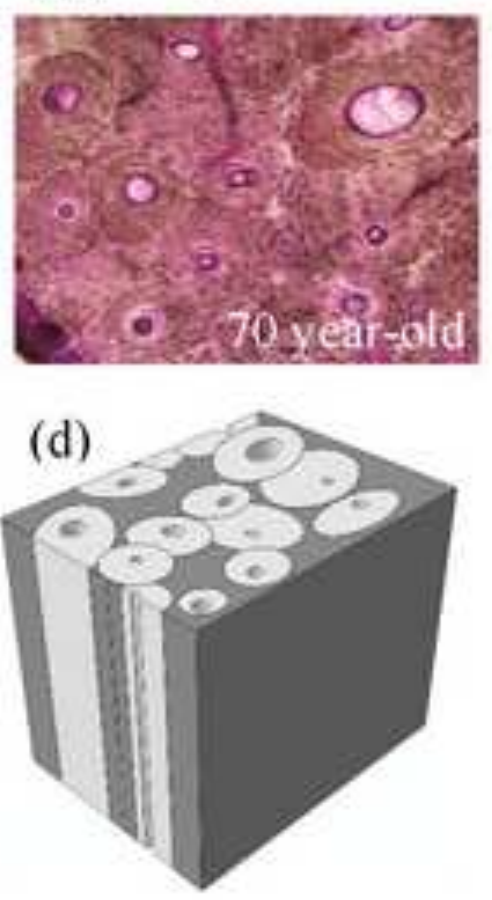
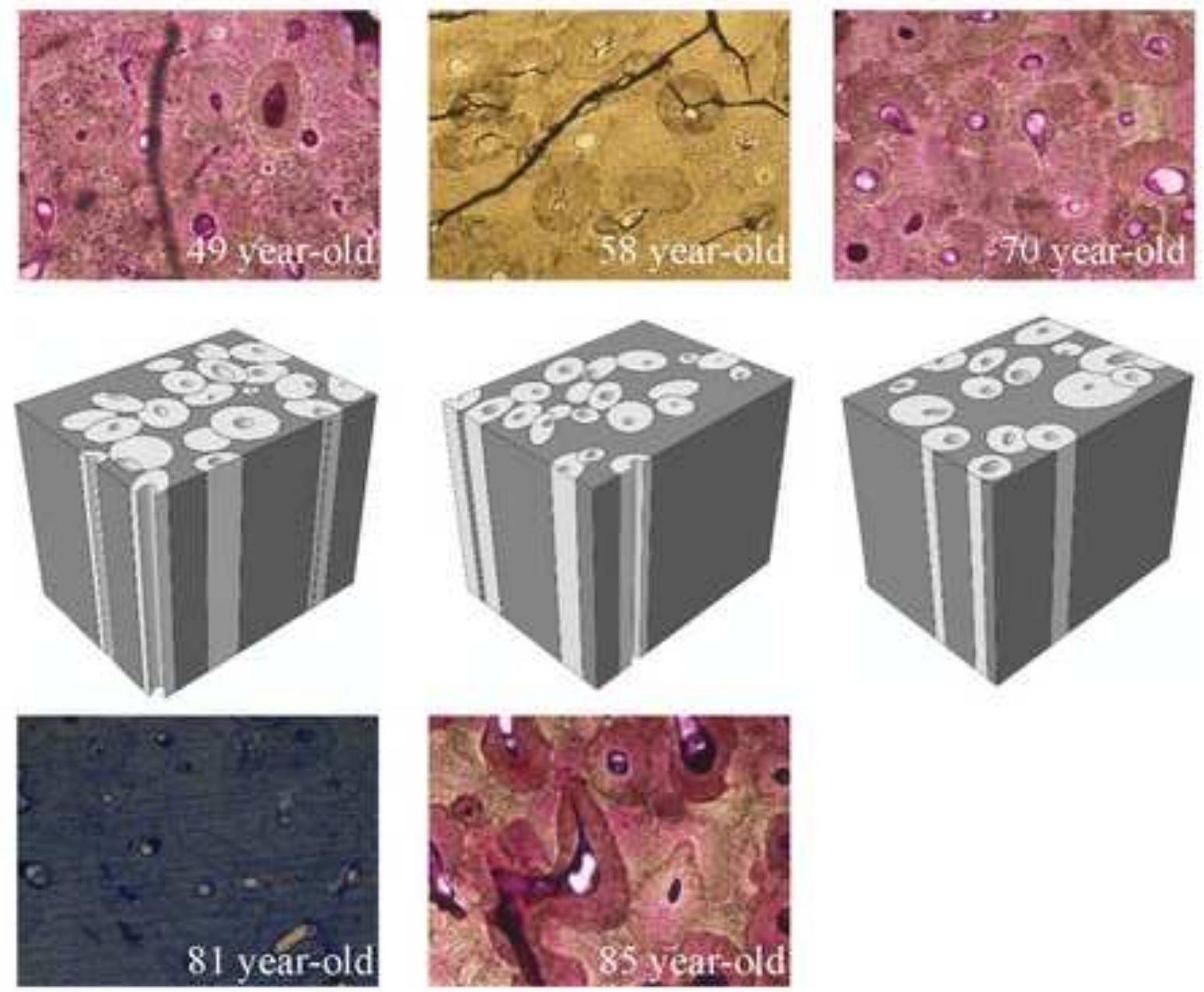
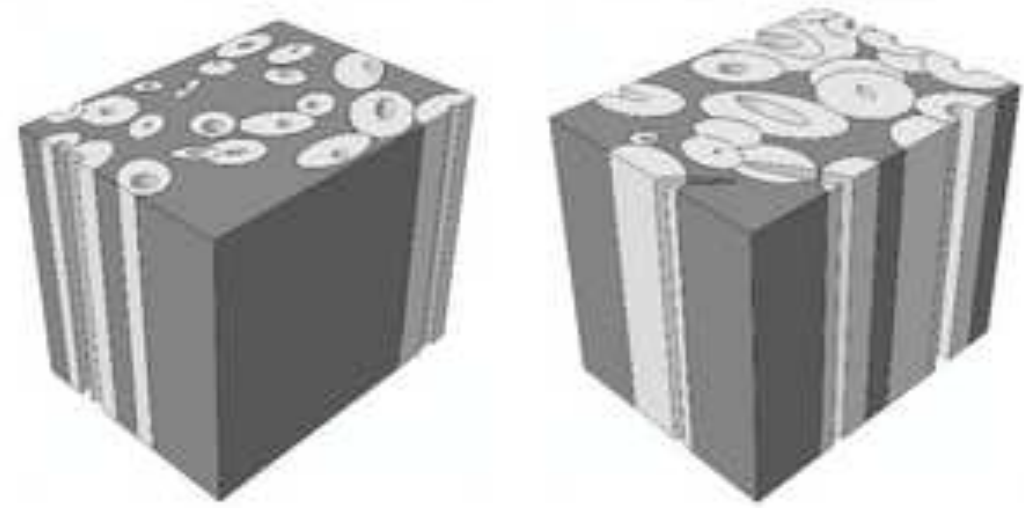


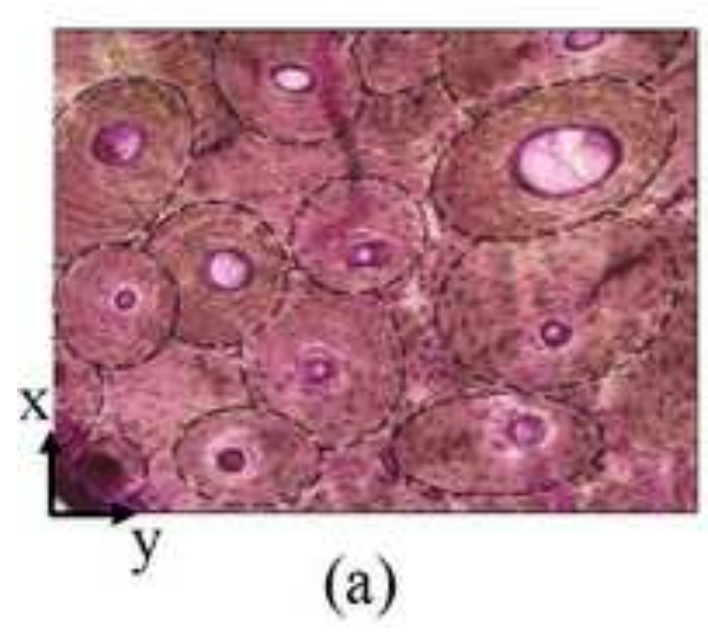

(a)

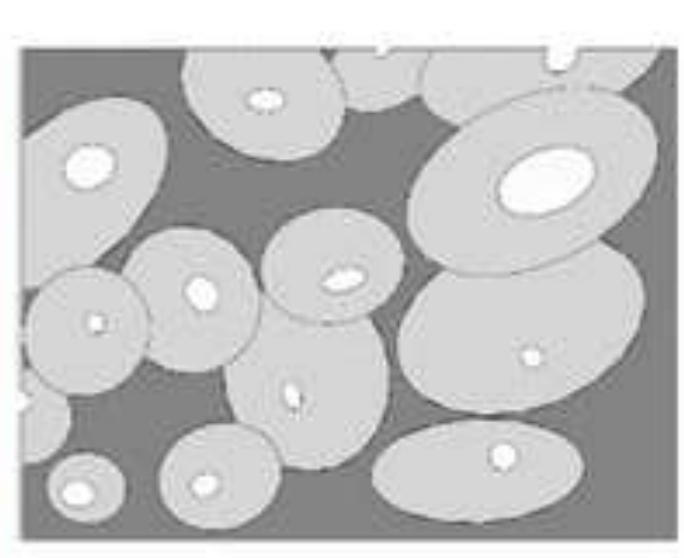

(b)

(b)

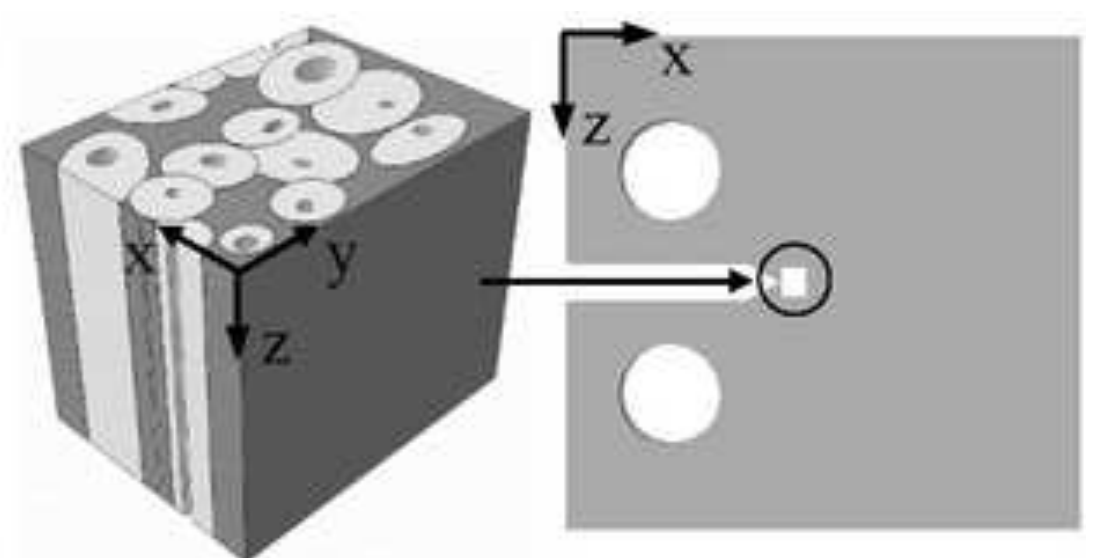

(c)

(d)

( 


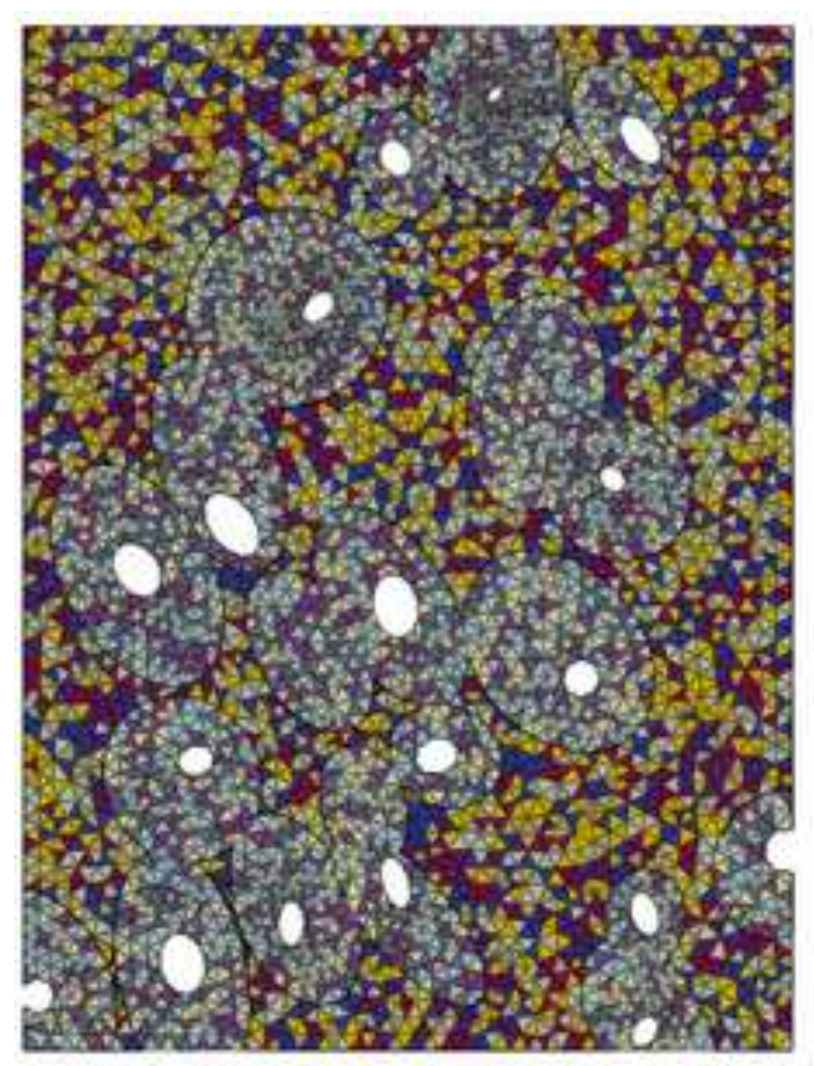

(a)

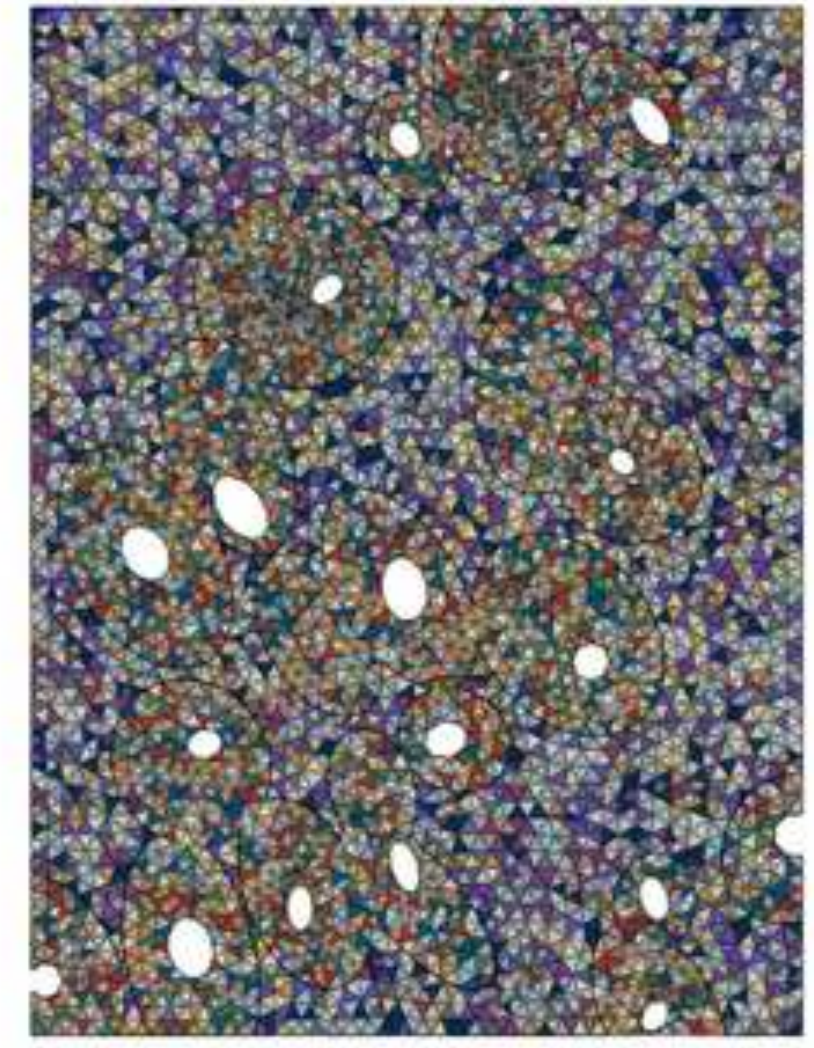

(b)

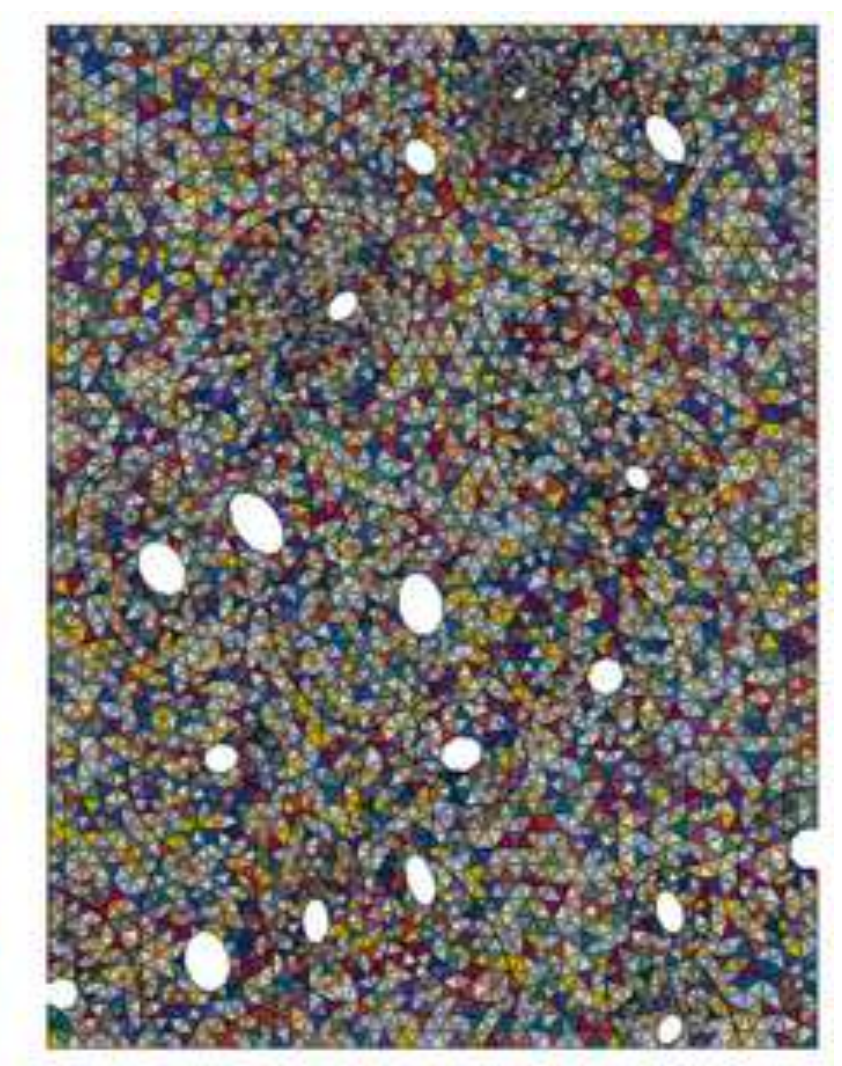

(c) 
Figure 4

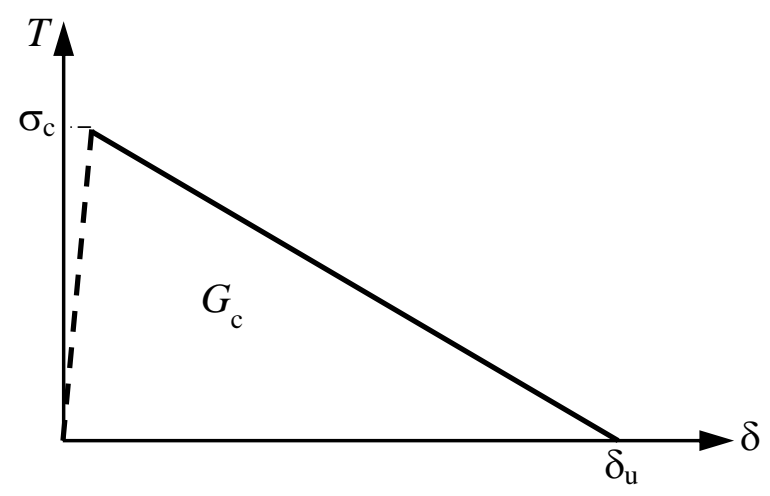


Figure 5

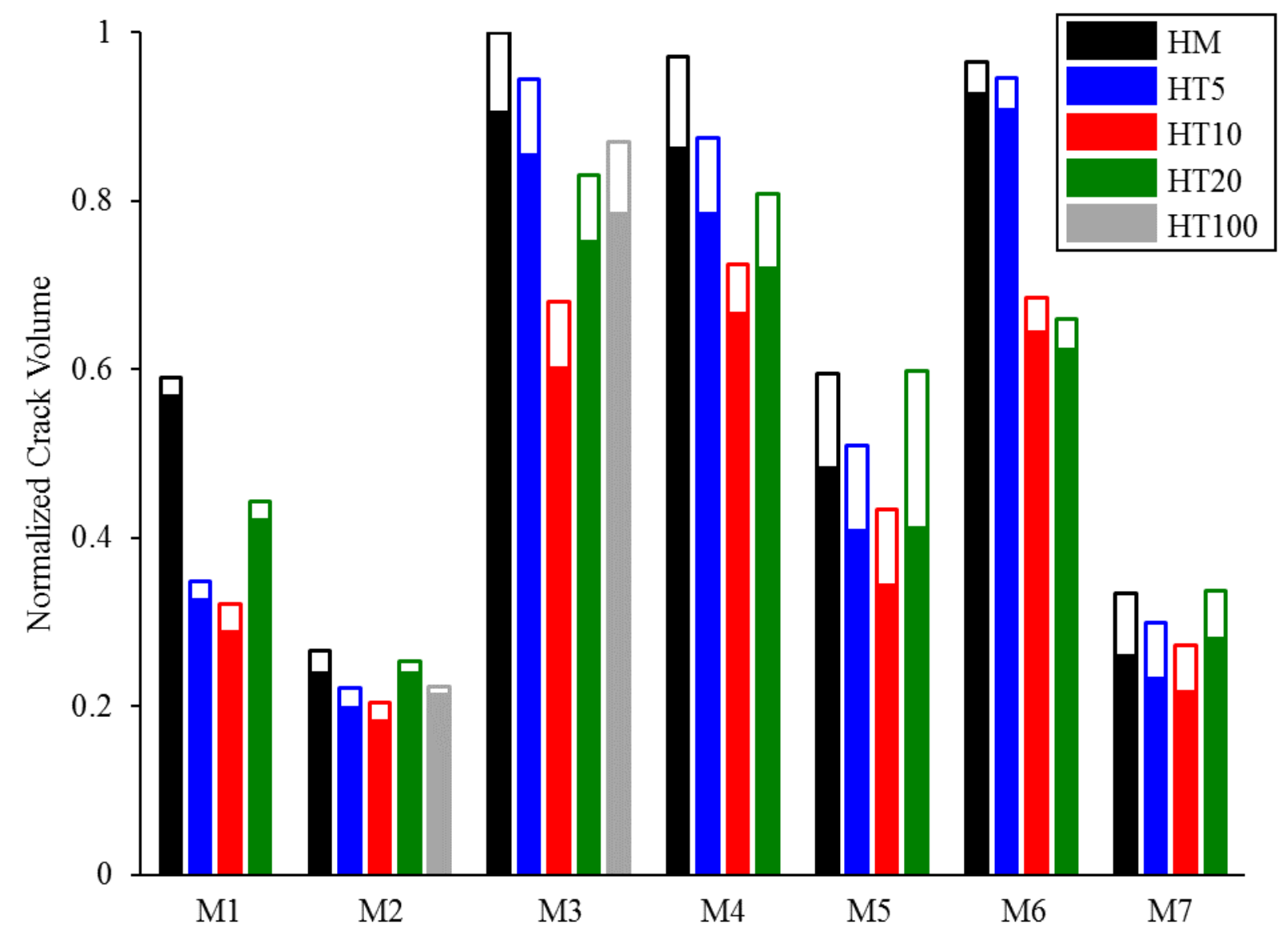


Figure 6

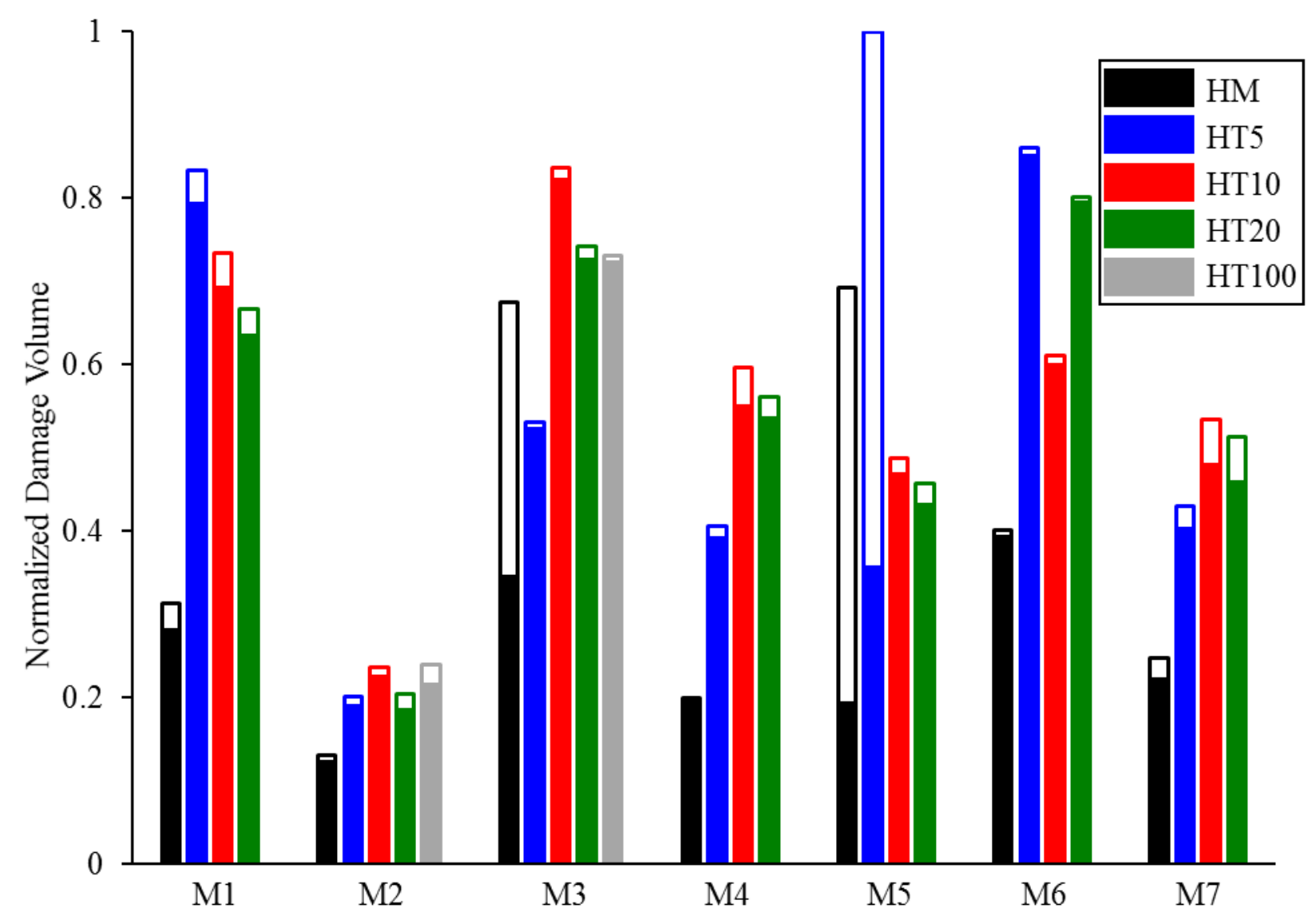


(a)

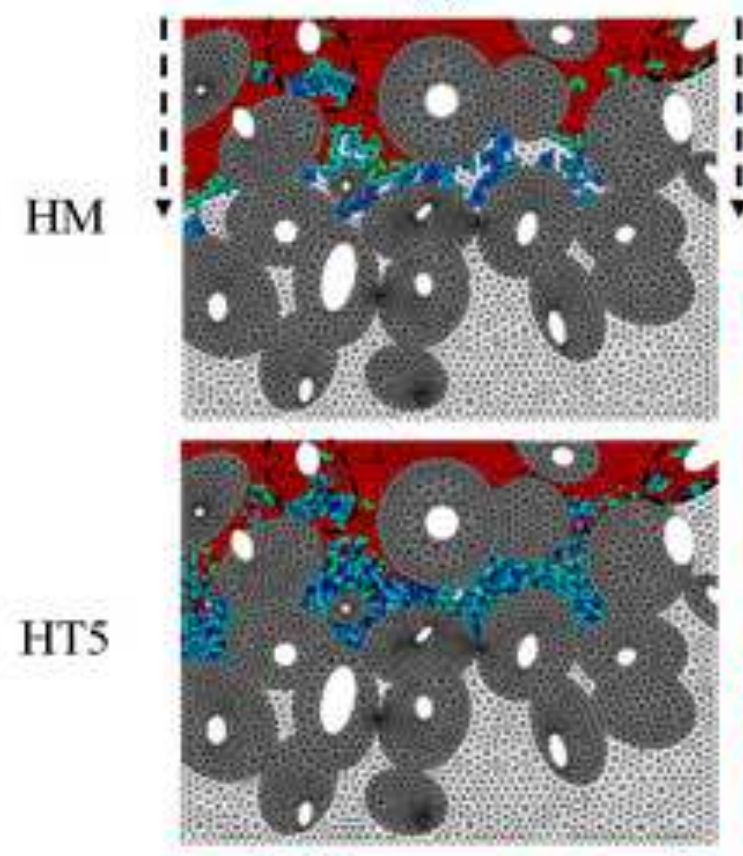

HT10

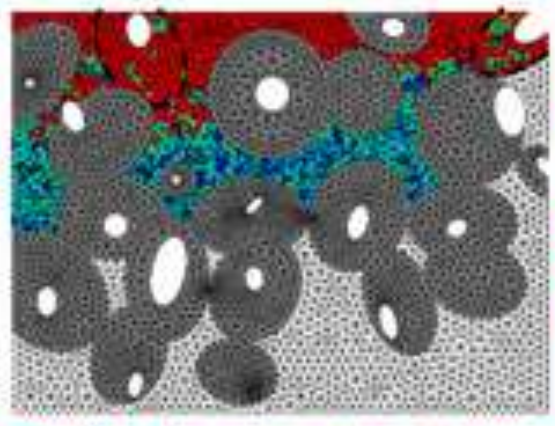

HT20

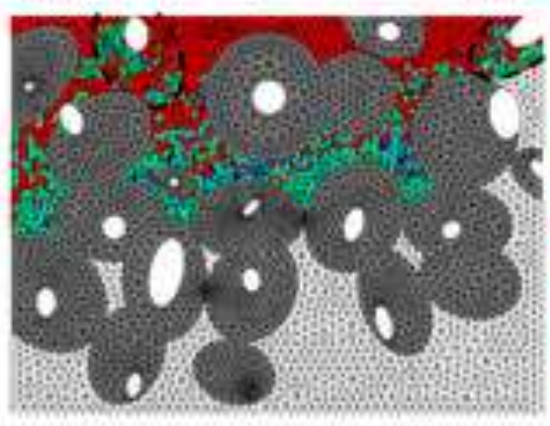

HT100

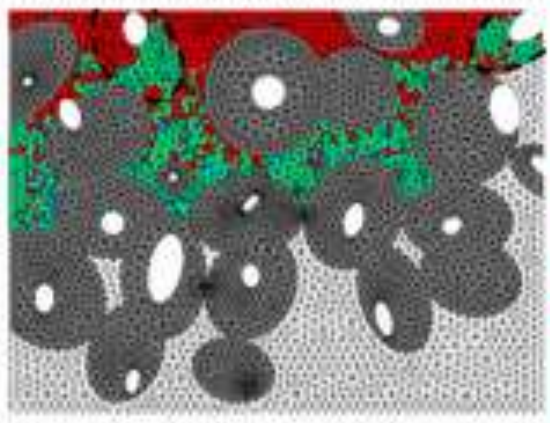

(b)

(c)
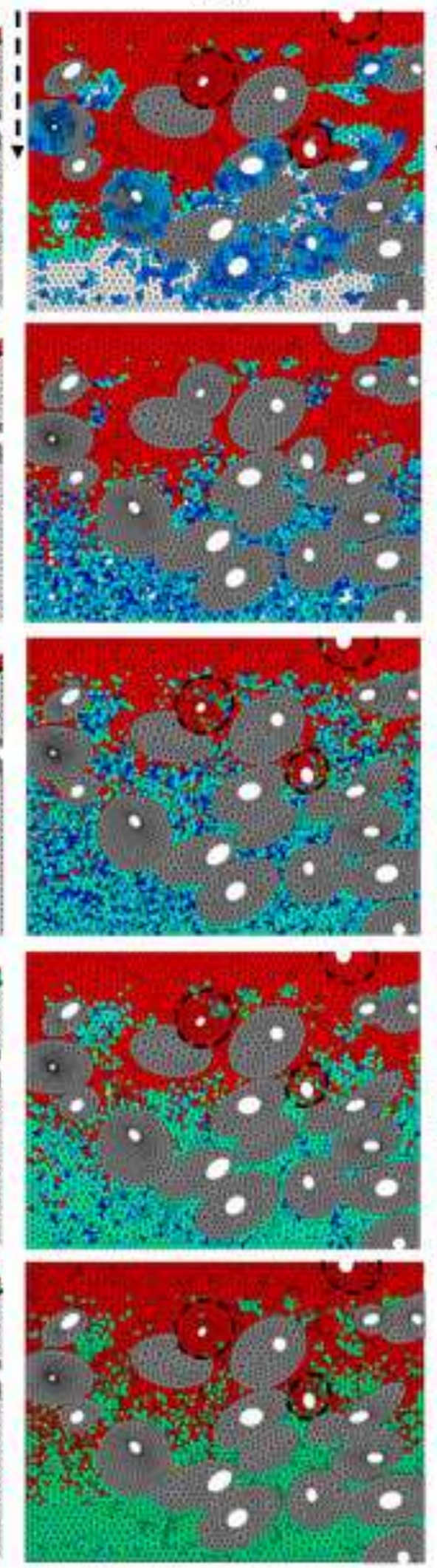
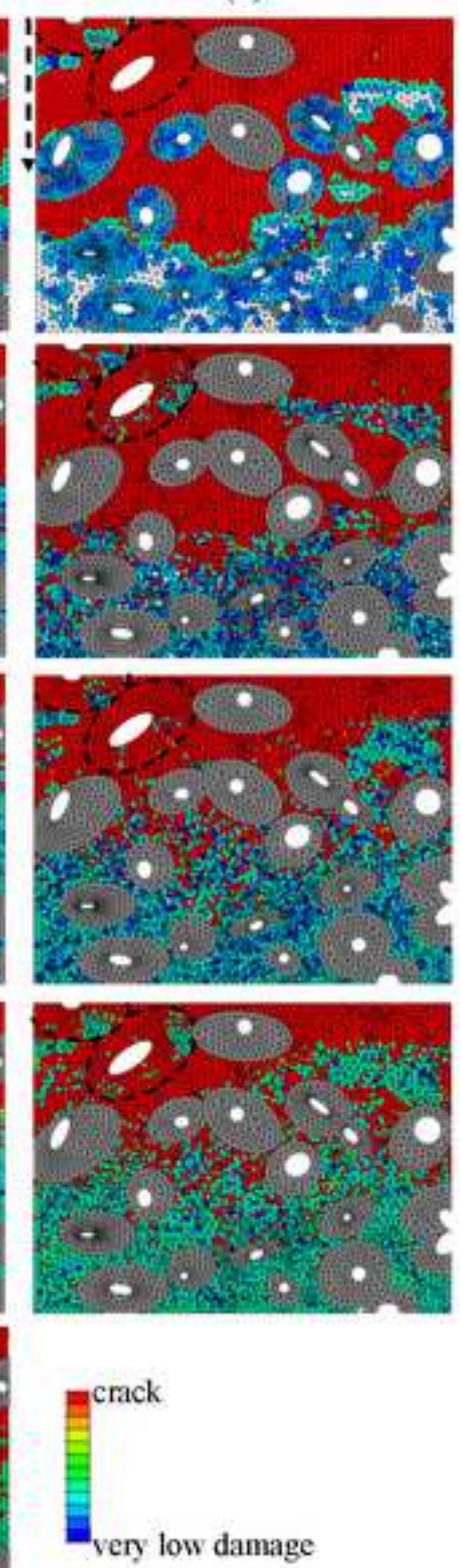
(b)
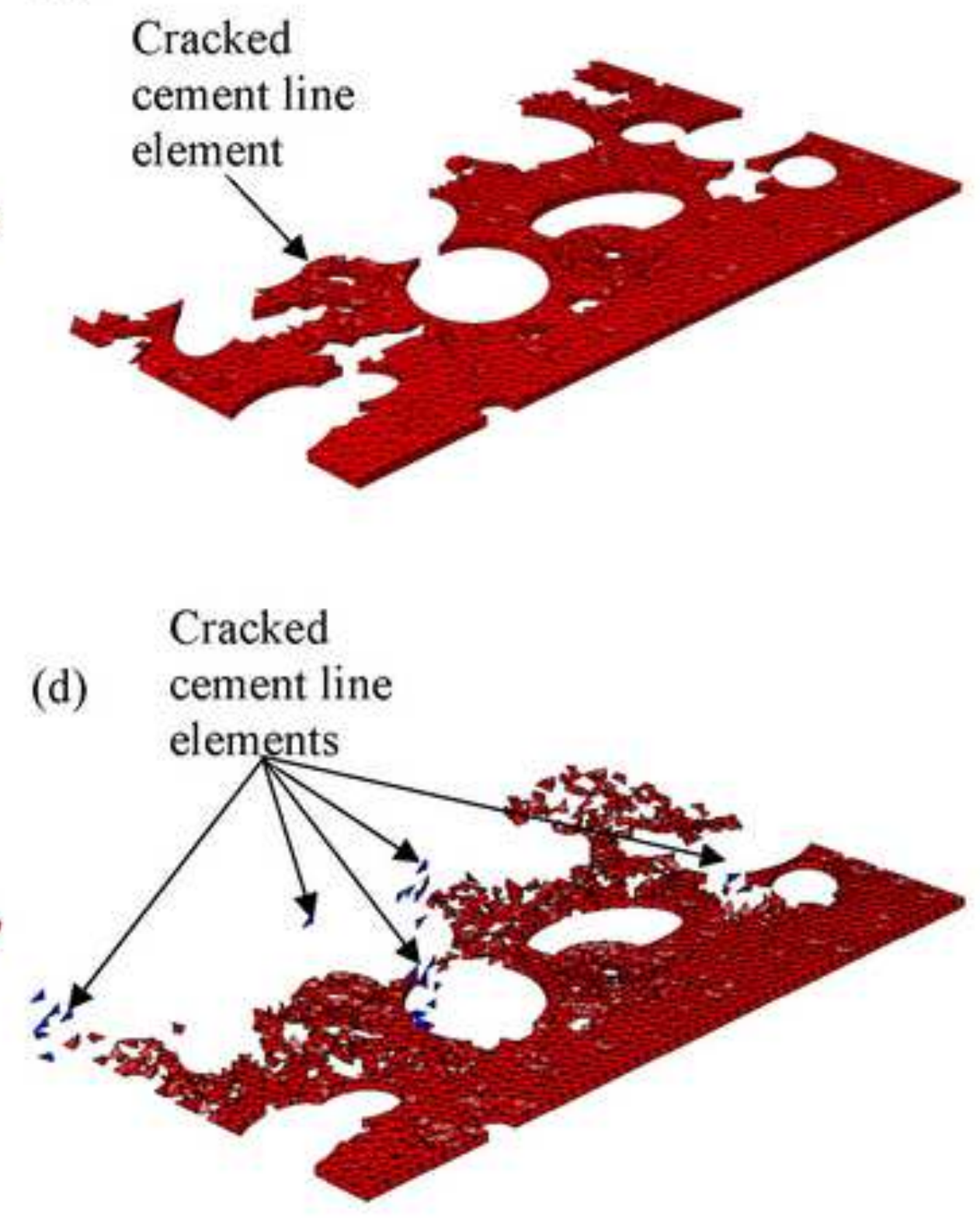
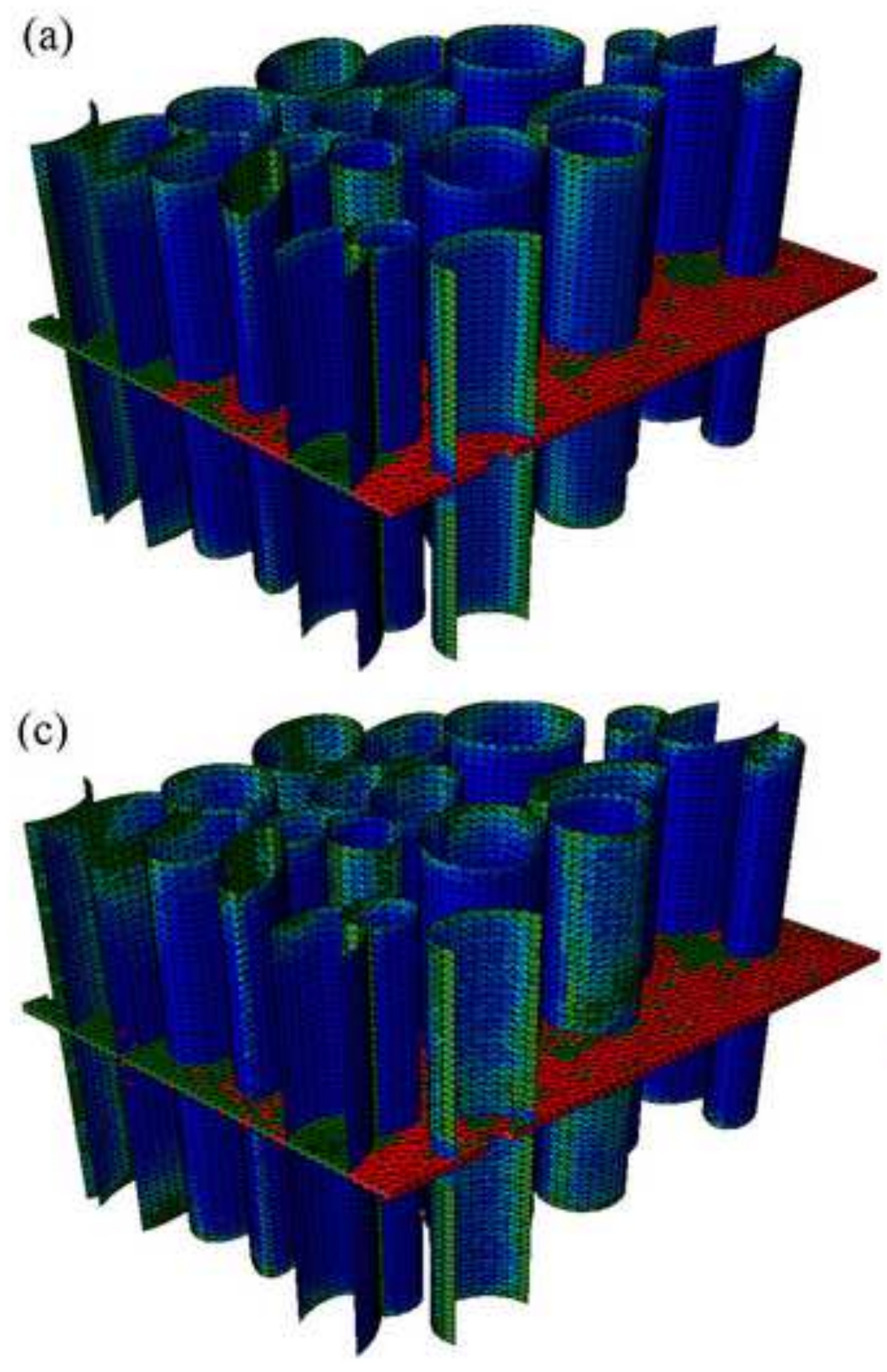

(a)

(c)
Cracked

(d) cement line

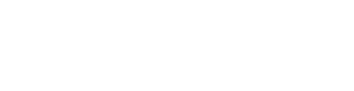


Figure 9

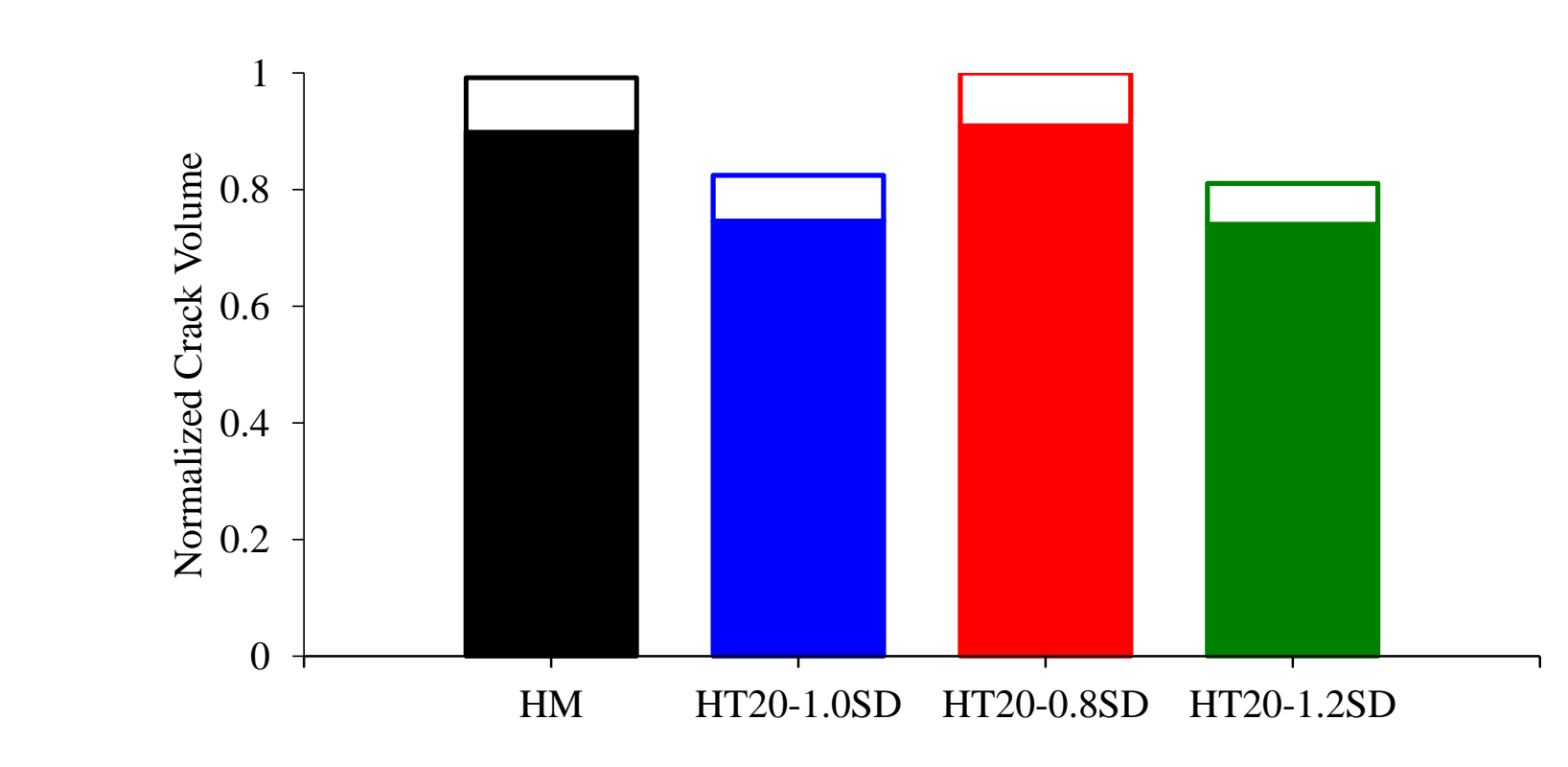

gure 9

.

